\title{
Four layer bandage compared with short stretch bandage for venous leg ulcers: systematic review and meta-analysis of randomised controlled trials with data from individual patients
}

\author{
Susan O'Meara, research fellow, ${ }^{1}$ Jayne Tierney, head of meta-analysis group, ${ }^{2}$ Nicky Cullum, deputy head of \\ department (research), ${ }^{1}$ J Martin Bland, professor of health statistics, ${ }^{1}$ Peter J Franks, professor of health \\ sciences, ${ }^{3,4}$ Trevor Mole, statistics manager, ${ }^{5}$ Mark Scriven, consultant vascular surgeon ${ }^{6}$
}

\section{'Department of Health Sciences, University of York, York Y010 5DD \\ ${ }^{2}$ Meta-analysis Group, MRC Clinical Trials Unit, London NW1 2DA \\ ${ }^{3}$ Centre for Research and Implementation of Clinical Practice, London SW15 5ES \\ ${ }^{4}$ Faculty of Medicine, Division of Nursing and Healthcare, University of Glasgow, Glasgow G12 8LW \\ ${ }^{5}$ Smith and Nephew Wound Management, Hull HU3 2BN \\ ${ }^{6}$ Department of Vascular Surgery, Birmingham Heartlands Hospital, Birmingham B9 5SS \\ Correspondence to: S O'Meara smo4@york.ac.uk}

Cite this as: $B M J$ 2009;338:b1344 doi:10.1136/bmj.b1344

\section{ABSTRACT}

Objective To compare the effectiveness of two types of compression treatment (four layer bandage and short stretch bandage) in people with venous leg ulceration.

Design Systematic review and meta-analysis of patient level data.

Data sources Electronic databases (the Cochrane Central Register of Controlled Trials, the Cochrane Wounds Group Specialised Register, Medline, Embase, CINAHL, and National Research Register) and reference lists of retrieved articles searched to identify relevant trials and primary investigators. Primary investigators of eligible trials were invited to contribute raw data for re-analysis.

Review methods Randomised controlled trials of four layer bandage compared with short stretch bandage in people with venous leg ulceration were eligible for inclusion. The primary outcome for the meta-analysis was time to healing. Cox proportional hazards models were run to compare the methods in terms of time to healing with adjustment for independent predictors of healing. Secondary outcomes included incidence and number of adverse events per patient.

Results Seven eligible trials were identified (887 patients), and patient level data were retrieved for five (797 patients, $90 \%$ of known randomised patients). The four layer bandage was associated with significantly shorter time to healing: hazard ratio ( $95 \%$ confidence interval) from multifactorial model based on five trials was 1.31 (1.09 to 1.58), $\mathrm{P}=0.005$. Larger ulcer area at baseline, more chronic ulceration, and previous ulceration were all independent predictors of delayed healing. Data from two trials showed no evidence of a difference in adverse event profiles between the two bandage types.

Conclusions Venous leg ulcers in patients treated with four layer bandages heal faster, on average, than those of people treated with the short stretch bandage. Benefits were consistent across patients with differing prognostic profiles.

\section{INTRODUCTION}

Venous leg ulcers are a common and recurring chronic wound caused by damage to the veins and consequent high venous pressure. ${ }^{1}$ The estimated lifetime prevalence for leg ulceration in developed countries is $1 \%$ and the point prevalence is $0.1-0.2 \%$. Prevalence increases with age and is higher among women. ${ }^{2}$ The UK Healthcare Commission has estimated that currently leg ulcer care costs the NHS $£ 300-600 \mathrm{~m}$ (€330$661 \mathrm{~m}, \$ 447-895 \mathrm{~m}$ ) a year. ${ }^{3}$ A substantial proportion of the costs is attributable to nursing time. ${ }^{45}$ These ulcers are also associated with increased costs and reduced health related quality of life for patients. ${ }^{46-8}$

Compression bandaging is thought to assist ulcer healing by reducing distension in the leg veins and accelerating venous blood flow. ${ }^{1}$ A previous systematic review of published trial level data concluded that compression was more effective in healing venous leg ulcers than no compression, multi-layered systems were more effective than single layer systems, and high compression was more effective than low compression, but no clear differences in effectiveness were detected between different types of high compression..$^{9}$

The four layer bandage and the short stretch bandage are examples of high compression (defined as ankle sub-bandage pressure $35-40 \mathrm{~mm} \mathrm{Hg}$ ). Such systems are deemed to deliver the optimum therapeutic effect in eligible patients but are contraindicated in people with clinically significant arterial disease. The four layer bandage (an elastic system), the standard method in the United Kingdom, comprises orthopaedic wool, crepe bandage, elastic bandage, and a final cohesive retaining layer. All layers are applied from toes to knee and normally require weekly renewal but can be changed more often if necessary. The short stretch system, used as standard treatment in mainland Europe and Australia, is an inelastic bandage, meaning that it has minimal extensibility (or "give") when handled. An orthopaedic wool layer is 
Total studies identified by search $(n=128)$ One unpublished study identified after main searches

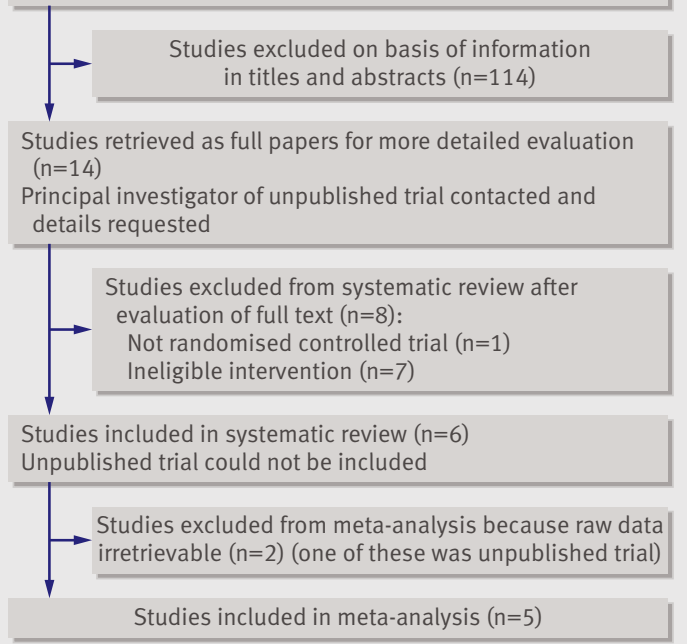

Fig 1 | Search strategy used for CENTRAL

covered by the bandage at full stretch to create a rigid casing around the limb that generates resistance against calf muscles and other tissues with reapplication every few days. ${ }^{1}$ The short stretch bandage has the advantage of being washable and reusable. ${ }^{10}$ Findings from a large clinical trial of compression treatment, however, indicated that few patients laundered and reused their short stretch bandages. ${ }^{\mathrm{w} 1}$ The four layer bandage, commonly available as a proprietary kit, is designed to be discarded after a single use.

A previous systematic review included two small trials that compared four layer and short stretch bandages. ${ }^{\text {w2 w3 }}$ At the time of the review, the only published trial level data available for both trials were the proportions of ulcers healed at three months, suggesting no significant differences between the two methods. One of the trials additionally reported healing rates at one year and time to healing, with no significant differences observed between bandage types. ${ }^{\text {w3 }}$ Estimates of the frequency of wound healing at fixed time points are less informative than time to healing as the latter has major implications for patients' health related quality of life and use of health service resources. A further limitation of the published trial level data was the inability to investigate subgroups of patients who might benefit differentially from treatment. We therefore carried out a systematic review and meta-analysis based on individual patient data to compare the effects of four layer bandage and short stretch bandage on time to healing of venous leg ulcers, taking account of prognostic factors. Our methods were based on those developed by the Cochrane Individual Patient Data Meta-analysis Methods Group. ${ }^{11}$

\section{METHODS}

All methods were prespecified in the systematic review and meta-analysis protocol (available on request).

\section{Study selection criteria}

Randomised controlled trials of four layer bandage compared with short stretch bandage for the treatment of venous leg ulcers were eligible for inclusion.

\section{Identification of trials}

In December 2005 we searched the Cochrane Register of Controlled Trials (CENTRAL) and the Cochrane Wounds Group Specialised Register with no restrictions applied to date, country, or language of publication. To cover the time lag between studies being indexed on primary sources and being listed on the Cochrane databases, we undertook additional searches for Medline, Embase, and CINAHL (20025). We searched the National Research Register to identify ongoing trials. All database searches were updated in March 2008. Figure 1 shows the search strategy. Initially, search terms were developed for CENTRAL and were adapted for use in the other databases. We examined the reference lists of eligible trials for additional relevant evaluations and asked collaborating trialists to provide details of any others known to them.

Two reviewers (SO'M and $\mathrm{NC}$ ) independently decided on study selection with disagreements resolved by discussion.

\section{Data collection and end points}

Authors of eligible trials were contacted initially during early 2005 and invited to collaborate in the meta-analysis. In cases of non-response, reminder messages were sent, and if necessary these were followed up with postal and telephone correspondence. We eventually established contact with authors of all relevant trials. We asked trialists to provide anonymised baseline and outcome data for each randomised patient, including those excluded from their own analyses, to maintain randomised groups and to provide as complete a dataset as possible for the meta-analysis. Baseline data included sex, age, primary or recurrent ulceration, ulcer duration, ulcer area, ulcer diameter, appearance of wound bed, ulcer infection, ankle brachial pressure index (ABPI), ankle circumference, ankle mobility, patient mobility, and history of comorbidities such as deep vein thrombosis. Outcome variables included healing status (that is, healed or not), date of healing, recurrence status, date of recurrence, ulcer area at follow-up points during the trial, and adverse events. In addition, the trialists were asked to provide date of randomisation, allocated treatment, date of last follow-up, and details of exclusion from analysis. All data were systematically checked for completeness, duplication, consistency, feasibility, and integrity of randomisation. ${ }^{11}$ Queries were resolved by discussion with the relevant trialist. Each included trial was assessed for adequacy of randomised sequence generation, allocation concealment, and blinding with methods based on the Cochrane Collaboration's tool for assessing risk of bias in clinical trials. $^{12}$ 
Time to healing was defined as the time from the date of randomisation to the date of healing, with healing defined as complete epithelialisation of the reference wound. Wounds were examined at least once a week in all trials. Data for patients with ulcers not healing within the trial period were censored on the date of last follow-up. Time to ulcer recurrence was defined as the time interval between healing and recurrence.

\section{Statistical analysis}

The patient was both the sampling unit and the unit of analysis. ${ }^{13}$ In cases where patients had multiple wounds included in the trial, we selected the largest for inclusion in the meta-analysis. Analyses were undertaken on an intention to treat basis (that is, according to randomised allocation group with inclusion of all patients as the aim). Imputation was not undertaken for missing data.

The primary outcome was time to healing. To provide a simple descriptive measure of this outcome, we performed a preliminary (unadjusted) analysis by generating non-stratified Kaplan-Meier survival curves for both treatment groups. The dependent variable was time to healing in days, the event was a healed ulcer, and the factor was bandage type.

Next, we generated a Cox proportional hazards model with time to healing in days as the dependent variable, healing as the event, and bandage type as a covariate. This preliminary model did not include adjustment for baseline characteristics. The main, formal, preplanned analysis entailed a Cox proportional hazards model as described above with additional covariates of sex, age, primary or recurrent ulceration, ulcer duration, ulcer area, ulcer diameter, appearance of wound bed, ulcer infection, ankle brachial pressure index, ankle circumference, ankle mobility, patient's mobility, and history of comorbidities-for example, deep vein thrombosis. Covariates found to be significant at the $5 \%$ level in univariate analyses were entered simultaneously into the model. We then used a backward elimination method to generate hazard ratio estimates of treatment effect. The model was extended to include tests of statistical interaction between type of bandage and baseline characteristics. To take account of any differences in healing rate between study centres, we entered centres into the model as strata. This automatically included trial as strata also as no centre was in more than one trial. To check that stratifying by study centre was the correct approach, we ran two preliminary Cox models including, firstly, bandage type and trial and, secondly, bandage type, trial, and centre as predictors. These models generated identical estimates of treatment effect and so we ran all the above Cox models stratifying only by study centre.

We carried out checks to assess the proportional hazards assumption; the linearity of the relation between the dependent variable and continuous covariates; whether time to healing was similar during early and late accrual ${ }^{14}$; and adequacy of model fit with regard to the relation between the number of events and the number of covariates included in each model. ${ }^{15}$

To generate a forest plot showing the relative contribution of each trial to the meta-analysis, we derived individual trial estimates from the individual patient data using Cox regression with covariate adjustment as per the final model as described above. These hazard ratio estimates were then combined to provide a visual display of the overall estimate of treatment effect. This secondary analysis allowed assessment of heterogeneity between trials, defined with the $\chi^{2}$ test (cut off $<10 \%$ for significance) and the $\mathrm{I}^{2}$ statistic (threshold of $>50 \%)^{1617}$

Secondary outcomes included time to ulcer recurrence; change in ulcer area at follow-up points during the trial as a predictor of time to healing; change in ulcer area during the trial as an outcome of treatment; and adverse events. Lack of data for the recurrence and ulcer area outcomes, however, meant that the planned analyses could not be undertaken and so these outcomes are not considered further here.

Adverse events were defined in two ways: as any adverse event or those considered by the original investigators to be related to the bandage. For each of these outcomes, we assessed the effect of bandage type on the incidence of adverse events using the odds ratio with associated 95\% confidence intervals. We compared the number of adverse events per patient for the two bandage systems using a weighted mean difference with associated 95\% confidence intervals. For all pooled analyses of adverse events, we defined statistical heterogeneity between individual trial estimates using the criteria described above. In the absence of significant heterogeneity, we generated a fixed effect model, otherwise we planned to use a random effects model.

Survival analyses were conducted with SPSS (version 15.0). The Kaplan-Meier plot was generated with Stata SE (version 10). Adverse event analyses and forest plots were generated with RevMan (version 4.2.8).

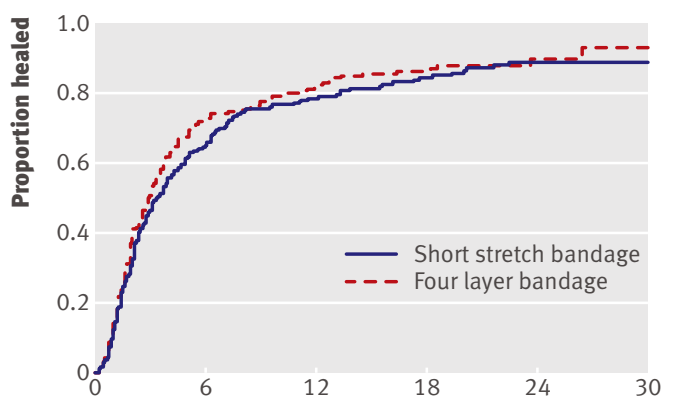

No at risk

Time (months)

bandage

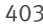

82

43

23

Four layer 394 bandage

Fig 2 | Kaplan-Meier survival curves (unstratified analysis) 


\section{RESULTS}

The search strategy retrieved 128 records of possible relevance. Of these, six trials were eligible for inclusion. $^{\text {w1 }}{ }^{\mathrm{w} 2 \mathrm{w} 3 \mathrm{w} 4 \mathrm{w} 5 \mathrm{w} 6}$ Four trials were conducted in the $\mathrm{UK}^{\mathrm{w} 1 \mathrm{w} 2 \mathrm{w} 3 \mathrm{w} 6}$ and the two others in mainland Europe. ${ }^{\mathrm{w} 4}$ w5 All trials were individually randomised and recruited participants with venous leg ulcers (defined by ankle brachial pressure index $\geq 0.8$ plus clinical history). Five trials included two treatment arms and compared the four layer bandage with the short stretch bandage. ${ }^{\mathrm{w} 1 \mathrm{w} 3 \mathrm{w} 4 \mathrm{w} 5 \mathrm{w} 6}$ One of these had a factorial design and included a randomised comparison of two types of primary dressing as well as of the two bandage systems. ${ }^{\text {w6 }}$ Another included an additional treatment arm that received a two layer paste and support bandage; we could not retrieve data for this trial because all records had been destroyed. ${ }^{\mathrm{w} 2}$

We identified an additional eligible unpublished trial opportunistically during a wound management conference. Contact with the primary investigator confirmed that the trial was small (40 patients), had terminated prematurely, and data were no longer available. The two trials for which data were unavailable recruited 83 eligible participants overall $(9.4 \%$ of known randomised patients). Table 1 shows details of all eligible trials. Table 2 shows patients' characteristics for the five trials with available individual patient data $(89.8 \%$ of known randomised patients) and suggests adequate balance of prognostic factors across treatment groups for the meta-analysis dataset.

Information gleaned from correspondence with trialists, trial reports, and the individual patient data suggested that all five trials have used adequate methods of randomisation and allocation concealment. Three trials used a simple method of randomisation. ${ }^{\mathrm{w} 4 \mathrm{w} 5 \mathrm{w} 6}$ One of these was unstratified, ${ }^{\text {w5 }}$ and two were stratified by ulcer area. ${ }^{\mathrm{w} 4}{ }^{\mathrm{w} 6}$ All used sealed envelopes to conceal allocation. The fourth trial used blocks of four for randomisation, stratified by ulcer area, and concealed allocation by using sealed envelopes. ${ }^{\text {w3 }}$ The fifth trial used permuted blocks stratified by study centre, previous ulceration, ulcer area, and ulcer duration, and concealed allocation by using a central telephone randomisation service. ${ }^{\mathrm{w}}$ Checks on the integrity of randomisation carried out on the individual patient data suggested that the balance of baseline variables across groups was satisfactory in each trial.

All five trials defined healing as complete epithelial cover of the ulcer site with non-blinded assessment by clinicians. ${ }^{\text {w1 w3 w4 w5 w6 }}$ In the largest trial, an investigator blinded to bandage allocation confirmed healing from photographs at the trial office; this was considered as an adequate procedure in terms of internal validity. ${ }^{\mathrm{w} 1}$

The characteristics of patients included in this metaanalysis seemed generally representative of those undergoing management of venous leg ulcers in outpatient or community settings. ${ }^{1819}$ The proportions of men and women reflected the relevant epidemiological evidence,$^{2}$ and there was an adequate spread of data for prognostic baseline variables such as ulcer area and ulcer duration. The data on ankle mobility and general mobility suggested that most patients were ambulant. Reported follow-up periods for trials ranged from three to 12 months. The overall median follow-up of patients who did not heal during the trial period was around 13 weeks (estimate derived from individual patient data).

Table 1| Summary of eligible trials on four layer bandage (4LB) compared with short stretch bandage (SSB) (all applied in accordance with manufacturers' instructions) in people with venous leg ulceration

\begin{tabular}{|c|c|c|c|c|c|c|}
\hline Study & $\begin{array}{l}\text { No }(\%) \text { of known } \\
\text { randomised } \\
\text { patients }\end{array}$ & $\begin{array}{l}\text { No of study } \\
\text { centres }\end{array}$ & Selection criteria & $\begin{array}{c}\text { Median follow-up } \\
\text { (weeks) for non-healed } \\
\text { patients, derived from } \\
\text { IPD }\end{array}$ & $\begin{array}{l}\text { No patients excluded from } \\
\text { trialists' analyses/ } \\
\text { reinstated for meta- } \\
\text { analysis }\end{array}$ & $\begin{array}{l}\text { Reasons for exclusion } \\
\text { from trialists' analyses }\end{array}$ \\
\hline $\begin{array}{l}\text { Unpublished trial } \\
\text { UK }\end{array}$ & $40(4.5)$ & Unknown & Unknown & NA & Unknown/NA & - \\
\hline $\begin{array}{l}\text { Duby et al,w2 } 1993 \text {, } \\
\text { UK }\end{array}$ & $43(4.8)$ & 1 & Venous leg ulcer; $A B P I \geq 0.9$ & NA & Unknown/NA & - \\
\hline Scriven et al, ${ }^{\text {w3 }} 1998$, UK & $53(6.0)$ & 1 & $\begin{array}{l}\text { Venous leg ulceration confirmed } \\
\text { with colour duplex scanning and } \\
A B P I \geq 0.8\end{array}$ & 4LB 13.0, SSB 17.3 & $4 \operatorname{LB} 1 / 0$, SSB $2 / 0$ & $\begin{array}{l}\text { 4LB no follow-up data; } \\
\text { SSB } 1 \text { no follow-up data, } \\
1 \text { died early in trial }\end{array}$ \\
\hline $\begin{array}{l}\text { Partsch et al,w4 } 2001 \text {, } \\
\text { Austria/Netherlands }\end{array}$ & $116(13.1)$ & 7 & $\begin{array}{l}\text { New episode of venous leg } \\
\text { ulceration; ulcer aetiology } \\
\text { confirmed by Doppler or clinical } \\
\text { history; ABPI } \geq 0.8\end{array}$ & 4LB 7.0, SSB 10.3 & Overall $4 / 0^{*}$ & $\begin{array}{l}3 \text { had no follow-up data, } 1 \\
\text { ineligible }\end{array}$ \\
\hline $\begin{array}{l}\text { Ukat et al, }{ }^{\text {w5 }} 2003 \text {, } \\
\text { Germany }\end{array}$ & $89(10.0)$ & 2 & Venous leg ulceration; $A B P I \geq 0.8$. & 4LB 11.9, SSB 12.0 & $4 \mathrm{LB} 0 / 0, \mathrm{SSB} 0 / 0$ & - \\
\hline Franks et al, ${ }^{\mathrm{w} 6} 2004$, UK & $159(18.0)$ & 12 & $\begin{array}{l}\text { Venous leg ulceration; ulcer } \\
\text { aetiology confirmed by clinical } \\
\text { history; } A B P I \geq 0.8 \text {. }\end{array}$ & 4LB 23.7, SSB 23.3 & $4 \mathrm{LB} 1 / 1, \mathrm{SSB} 2 / 2$ & All 3 ineligible \\
\hline Iglesias et al, ${ }^{\mathrm{w} 1} 2004$, UK & $387(43.6)$ & 9 & $\begin{array}{l}\text { Venous leg ulcer } \geq 1 \mathrm{~cm} \text { diameter; } \\
A B P I \geq 0.8 \text {. }\end{array}$ & 4LB 55.0, SSB 54.0 & $4 \mathrm{LB} 0 / 0, \mathrm{SSB} 0 / 0$ & - \\
\hline Total & $887(100)$ & 32 & - & 4LB 13.0, SSB 12.3 & Overall $10 / 3$ & - \\
\hline
\end{tabular}

$\mathrm{ABPI}=$ ankle brachial pressure index; $\mathrm{IPD}=$ individual patient data; $\mathrm{NA}=$ not applicable as unable to retrieve individual patient data;

*Breakdown per group not provided. 
Table 2 |Characteristics of patients from trials with available individual patient data. Figures are numbers (percentages) of patients unless stated otherwise

\begin{tabular}{|c|c|c|c|}
\hline Variable & $\begin{array}{l}\text { Four layer bandage } \\
\qquad(n=394)\end{array}$ & $\begin{array}{l}\text { Short stretch bandage } \\
\qquad(n=403)\end{array}$ & Total $(n=797)$ \\
\hline \multicolumn{4}{|l|}{ Sex: } \\
\hline Male & $151(38.3)$ & $166(41.2)$ & 317 (39.8) \\
\hline Female & $242(61.4)$ & $237(58.8)$ & $479(60.1)$ \\
\hline Not recorded & $1(0.3)$ & 0 & $1(0.1)$ \\
\hline \multicolumn{4}{|l|}{ Age (years): } \\
\hline Mean (SD) & $69.5(13.1)$ & $70.3(13.8)$ & $69.9(13.5)$ \\
\hline Median (range) & 71.8 (19-99) & $73.0(23-100)$ & $73.0(19-100)$ \\
\hline Not recorded & 0 & $1(0.3)$ & $1(0.1)$ \\
\hline \multicolumn{4}{|l|}{ Ulcer status: } \\
\hline First & $75(19.0)$ & $86(21.3)$ & $161(20.2)$ \\
\hline Recurrent & $287(72.8)$ & $286(71.0)$ & 573 (71.9) \\
\hline Not recorded & $32(8.1)$ & $31(7.7)$ & $63(7.9)$ \\
\hline \multicolumn{4}{|c|}{ Ulcer duration (months): } \\
\hline$\leq 1$ & $120(30.5)$ & $122(30.3)$ & $242(30.4)$ \\
\hline$>1-6$ & $157(39.8)$ & $173(42.9)$ & 330 (41.4) \\
\hline$>6-12.00$ & $41(10.4)$ & $40(9.9)$ & $81(10.2)$ \\
\hline$>12$ & $73(18.5)$ & $60(14.9)$ & $133(16.7)$ \\
\hline Not recorded & $3(0.8)$ & $8(2.0)$ & $11(1.4)$ \\
\hline \multicolumn{4}{|l|}{ Ulcer area $\left(\mathrm{cm}^{2}\right)$ : } \\
\hline Mean (SD) & $13.7(36.7)$ & $10.3(18.8)$ & $12.0(29.2)$ \\
\hline Median (range) & $4.3(0.2-378.3)$ & $4.3(0.4-143.9)$ & $4.3(0.2-378.3)$ \\
\hline Not recorded & $21(5.3)$ & $27(6.7)$ & $48(6.0)$ \\
\hline \multicolumn{4}{|l|}{ Presence of slough: } \\
\hline Non-sloughy & $110(27.9)$ & $128(31.8)$ & 238 (29.9) \\
\hline Sloughy & 199 (50.5) & $177(43.9)$ & $376(47.2)$ \\
\hline Not recorded & $85(21.6)$ & $98(24.3)$ & $183(23.0)$ \\
\hline \multicolumn{4}{|c|}{ Presence of granulation: } \\
\hline Non-granulating & $122(31.0)$ & $126(31.3)$ & $248(31.1)$ \\
\hline Granulating & $187(47.4)$ & $184(45.7)$ & $371(46.6)$ \\
\hline Not recorded & $85(21.6)$ & $93(23.1)$ & $178(22.3)$ \\
\hline \multicolumn{4}{|c|}{ Presence of epithelialising tissue: } \\
\hline Non-epithelialising & $226(57.4)$ & $221(54.8)$ & $447(56.1)$ \\
\hline Epithelialising & $29(7.4)$ & $32(7.9)$ & $61(7.7)$ \\
\hline Not recorded & $139(35.3)$ & $150(37.2)$ & $289(36.3)$ \\
\hline \multicolumn{4}{|c|}{ Ankle brachial pressure index: } \\
\hline Mean (SD) & $1.09(0.18)$ & $1.08(0.15)$ & $1.08(0.16)$ \\
\hline Median (range) & $1.05(0.76-2.00)$ & $1.06(0.75-1.70)$ & $1.06(0.75-2.00)$ \\
\hline Not recorded & $19(4.8)$ & $11(2.7)$ & $30(3.8)$ \\
\hline \multicolumn{4}{|l|}{ Ankle circumference: } \\
\hline Mean (SD) & $23.9(2.8)$ & $23.9(2.8)$ & $23.9(2.8)$ \\
\hline Median (range) & $24.0(16.2-34.0)$ & $24.0(16.0-33.0)$ & $24.0(16.0-34.0)$ \\
\hline Not recorded & $3(0.8)$ & $6(1.5)$ & $9(1.1)$ \\
\hline \multicolumn{4}{|l|}{ Ankle mobility: } \\
\hline Fully mobile & $289(73.4)$ & $294(73.0)$ & $583(73.1)$ \\
\hline Impaired & $103(26.1)$ & $104(25.8)$ & $207(26.0)$ \\
\hline Not recorded & $2(0.5)$ & $5(1.2)$ & $7(0.9)$ \\
\hline \multicolumn{4}{|l|}{ Patient mobility: } \\
\hline Fully mobile & $264(67.0)$ & $265(65.8)$ & $529(66.4)$ \\
\hline Impaired & $103(26.1)$ & $111(27.6)$ & 214 (26.9) \\
\hline Not recorded & $27(6.9)$ & $27(6.7)$ & $54(6.8)$ \\
\hline \multicolumn{4}{|c|}{ History of deep vein thrombosis: } \\
\hline No & $147(37.3)$ & $165(40.9)$ & $312(39.1)$ \\
\hline Yes & $52(13.2)$ & $46(11.4)$ & $98(12.3)$ \\
\hline Not recorded & $195(49.5)$ & $192(47.6)$ & 387 (48.6) \\
\hline
\end{tabular}

Data checking procedures showed a low number of errors, such as inconsistent dates, unfeasible values, and discrepancies between the individual patient data and the published trial report. These were all corrected through discussions with the relevant trialists, most being issues that merely required clarification. Ten patients were excluded from the original investigators' own analyses (1.1\% of known randomised patients), of whom three were reinstated for our meta-analysis (table 1). Data for the seven other patients were not available from the trialists.

The results of model checking procedures indicated that the proportional hazards assumption was upheld for all potentially predictive covariates. The only continuous covariate included in the final models - baseline ulcer surface area-was entered as a natural log transformation. Outcomes were similar for early and late accruals when we took into account differences between study centres in four trials assessed. ${ }^{\mathrm{w} 1 \mathrm{w} 4 \mathrm{w} 5 \mathrm{w} 6}$ To assess the adequacy of model fit, we assessed the number of events (that is, healings) against the number of covariates entered at the start of each model. At least 10 events per variable are required in logistic regression to reduce bias in regression coefficients. ${ }^{15}$ All models generated from the meta-analysis dataset met this recommendation.

\section{Time to healing}

\section{Preliminary analysis}

The median time to healing estimated from unstratified Kaplan-Meier survival analysis of all available patients $(\mathrm{n}=797)$ was 90 days for four layer bandage and 99 days for the short stretch bandage (fig 2).

\section{Main analysis}

An initial Cox proportional hazards model based on all five trials (797 patients, 20/797 cases dropped) was generated with time to healing (days) as the dependent variable, healing as the event, study centres as strata, and bandage type as the only covariate. The result of this unadjusted analysis indicated no significant difference between bandage types: hazard ratio 1.15, 95\% confidence interval 0.97 to $1.37 ; \mathrm{P}=0.11$.

The next Cox model (five trials, 797 patients, 75/797 cases dropped) included all significant covariates identified during univariate analyses: bandage type, patient's age, ulcer duration, ulcer area, and ankle mobility. We used a backward elimination method to remove covariates that became non-significant one at a time, until the model contained only those making a significant contribution. The final model contained type of bandage, ulcer duration, and ulcer area (table 3). The hazard ratio for bandage type was 1.31 (1.09 to $1.58 ; \mathrm{P}=0.005)$, indicating that the estimated probability of healing with four layer bandage was around 1.3 times that of healing with the short stretch bandage, assuming similar values for other covariates. There was significant evidence that larger ulcers $(\mathrm{P}<0.001)$ and ulcers of longer duration $(\mathrm{P}<0.001)$ predicted longer time to healing independently of one another and of treatment. The chance of healing was reduced 


\begin{tabular}{|c|c|c|c|c|c|c|c|}
\hline \multirow[b]{2}{*}{ Study } & \multicolumn{2}{|c|}{ No of patients } & \multirow[b]{2}{*}{$\begin{array}{l}\text { Log hazard } \\
\text { ratio (SE) }\end{array}$} & \multirow{2}{*}{\multicolumn{2}{|c|}{$\begin{array}{c}\text { Hazard ratio } \\
\text { (fixed effect) } \\
(95 \% \mathrm{Cl})\end{array}$}} & \multirow[b]{2}{*}{$\begin{array}{c}\text { Weight } \\
\text { (\%) }\end{array}$} & \multirow[b]{2}{*}{$\begin{array}{c}\text { Hazard ratio } \\
\text { (fixed effect) } \\
(95 \% \mathrm{Cl})\end{array}$} \\
\hline & $\begin{array}{l}\text { Short } \\
\text { stretch } \\
\text { bandage }\end{array}$ & $\begin{array}{c}\text { Four } \\
\text { layer } \\
\text { bandage }\end{array}$ & & & & & \\
\hline Scriven ${ }^{\text {w3 }}$ & 23 & 27 & $0.4380(0.4170)$ & & & 5.45 & 1.55 (0.69 to 3.51$)$ \\
\hline Partsch ${ }^{\mathrm{W}}$ & 59 & $53-$ & $-0.3470(0.2560)$ & 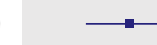 & - & 14.47 & 0.71 (0.43 to 1.17$)$ \\
\hline Ukat $^{\mathrm{w5}}$ & 45 & 44 & $0.7000(0.4540)$ & & - & 4.60 & 2.01 (0.83 to 4.91$)$ \\
\hline Franks ${ }^{w 6}$ & 84 & 75 & $0.4390(0.2720)$ & & $\longrightarrow$ & 12.81 & 1.55 (0.91 to 2.64$)$ \\
\hline Iglesias $^{\mathrm{w1}}$ & $1 \quad 192$ & 195 & $0.3490(0.1230)$ & & $\rightarrow-$ & 62.67 & $1.42(1.11$ to 1.80$)$ \\
\hline \multirow[t]{3}{*}{ Total } & 403 & 394 & & & $<$ & 100.00 & 1.31 (1.09 to 1.58$)$ \\
\hline & & & & 0.2 & 2 & 5 & \\
\hline & & & & $\begin{array}{l}\text { Favours } \\
\text { short stretch } \\
\text { bandage }\end{array}$ & $\begin{array}{l}\text { Fav } \\
\text { four } \\
\text { band }\end{array}$ & & \\
\hline
\end{tabular}

Fig 3 Hazard ratio plot for time to healing with pooled and individual estimates adjusted for baseline ulcer area and ulcer duration

by a factor of 0.44 for each 10 -fold increase in area. We categorised baseline ulcer duration into $\leq 1 \mathrm{month},>$ 1- 6 months, $>6-12$ months, and $>12$ months. The data suggest that the hazard of healing was reduced for each step up to a longer duration interval. We found no significant interactions between bandage and baseline ulcer area and bandage and ulcer duration. Figure 3 illustrates the relative contribution of each trial to the meta-analysis, showing pooled and individual trial hazard ratio estimates derived from individual patient data and adjusted for baseline ulcer area and baseline ulcer duration (heterogeneity between trials: $\chi^{2}$ test $\mathrm{P}=0.11, \mathrm{I}^{2}=47.7 \%$ ).

We re-ran the analysis on a subset of four trials (747 patients, 83/747 dropped) for which additional covariates were available: primary or recurrent ulceration and patients' mobility. ${ }^{\mathrm{w} 1 \mathrm{w} 4 \mathrm{w} 5 \mathrm{w} 6}$ The final model contained bandage type, ulcer area, ulcer duration, and primary or recurrent ulceration. The estimated hazard ratio for type of bandage was similar to the model based on five trials: $1.29,1.06$ to $1.57 ; \mathrm{P}=0.011$. The model suggested that larger ulcers $(\mathrm{P}<0.001)$, ulcers of longer duration $(\mathrm{P}<0.001)$, and previous ulceration $(\mathrm{P}<0.005)$ were independent predictors of longer time to healing (table 4). We found no significant interactions between bandage and baseline ulcer area, bandage and baseline ulcer duration, and bandage and primary or recurrent ulceration. The level of statistical heterogeneity between trials did not attain our prespecified level of significance $\left(\chi^{2}\right.$ test $\left.\mathrm{P}=0.11, \mathrm{I}^{2}=50.0 \%\right)$.

Table 3 | Final model based on five trials. Regression coefficients ( $\beta$ ) with standard errors (SE) and hazard ratios (HR) with $95 \%$ confidence intervals

\begin{tabular}{lccc} 
Variable & $\beta(\mathrm{SE})$ & HR $(95 \% \mathrm{Cl})$ & P value \\
Bandage & $0.27(0.10)$ & $1.31(1.09$ to 1.58$)$ & 0.005 \\
\hline Duration category (months): & & & \\
\hline Overall & - & $0.89(0.71$ to 1.11$)$ & 0.001 \\
\hline $1.01-6.0 \vee 0-1$ & $-0.12(0.11)$ & $0.59(0.40$ to 0.85$)$ & 0.005 \\
\hline $6.01-12.0 \vee 0-1$ & $-0.53(0.19)$ & $0.35(0.24$ to 0.50$)$ & $<0.001$ \\
\hline$>12$ v $0-1$ & $-1.07(0.19)$ & $0.70(0.64$ to 0.77$)$ & $<0.001$ \\
\hline Log $_{\mathrm{e}}$ ulcer area & $-0.36(0.05)$ & &
\end{tabular}

\section{Adverse events}

Two trials provided data on adverse events. ${ }^{\mathrm{w} 1 \mathrm{w} 6}$ For incidence of any type of adverse event, the pooled odds ratio (fixed effect) was 1.15 (95\% confidence interval 0.81 to $1.62 ; \mathrm{P}=0.43$ ) (fig 4 ), providing no evidence of a difference between bandage types. The two trials differed in their definitions of adverse events related to bandaging. One trial coded events such as maceration, allergic reaction, eczema of periulcer skin, and infection as bandage related. ${ }^{\text {w1 }}$ Another trial, which compared primary dressings as well as bandages, attributed these events to the former. ${ }^{\text {w6 }}$ In view of this difference, we did not pool data. We estimated odds ratios for each trial individually and neither showed a significant difference between groups: 1.41 (0.94 to $2.11)^{\mathrm{w} 1}$ and 0.78 (0.30 to 2.04$){ }^{\mathrm{w} 6}$

Analysis of the number of all types of adverse event per patient did not show a difference between the two bandage systems: pooled weighted mean difference (random effects) 0.21 (95\% confidence interval -0.27 to $0.68 ; \mathrm{P}=0.39$ ) (fig 5). Again, we did not pool data for adverse events related to bandaging for the reasons mentioned above. We found no significant differences between groups for the trials individually $(0.45(-0.11$ to 1.01$)^{\mathrm{w} 1}$ and $\left.-0.04(-0.17 \text { to } 0.09)^{\mathrm{w} 6}\right)$.

\section{DISCUSSION}

When compared with short stretch bandage, the four layer bandage increases the chance of healing by around 30\% when independent prognostic factors are taken into account. The change in hazard ratio estimate when we included the prespecified covariates in the model is to be expected because the covariates are significant predictors of time to healing. ${ }^{20}$ We therefore consider that the estimate adjusted for covariates generated by this meta-analysis provides the best unbiased estimate to date of the comparative effects of four layer and short stretch bandages on healing of venous leg ulcers. Estimates from tests of statistical interaction indicated that the benefit of four layer bandaging is consistent across patients with differing prognostic profiles. The largest trial incorporated a rigorous economic analysis and concluded that the four layer system was the dominant treatment strategy (that is, it had lower costs with greater health benefits). The cost per ulcer healed was estimated by taking account of nurse and doctor visits (both home and clinic based), visits to hospital, and costs of bandages and was based on Kaplan-Meier estimates of time to healing. The mean (95\% confidence interval) annual costs of treatment were $£ 1298.41$ ( $£ 1187.83$ to $£ 1471.89$ ) for the four layer bandage and $£ 1525.73$ ( $£ 1373.92$ to $£ 1716.66$ ) for the short stretch bandage, a difference of $£ 227.32$ (£16.53 to £448.30) (costs for year 2001). ${ }^{\mathrm{w} 1}$ Only two trials provided adequate data on adverse events. ${ }^{\text {w1 } 6}$ The available data represented 546 out of a total of 887 patients known to be eligible for inclusion in the meta-analysis $(62 \%$ of total randomised patients as shown in table 1).

Findings from our meta analysis are consistent with those from prognostic studies in suggesting that 
Table $4 \mid$ Final model based on four trials. Regression coefficients ( $\beta$ ) with standard errors (SE) and hazard ratios (HR) with $95 \%$ confidence intervals

\begin{tabular}{lclc} 
Variable & $\beta(\mathrm{SE})$ & HR $(95 \%$ Cl for HR) & P value \\
Bandage & $0.25(0.10)$ & $1.29(1.06$ to 1.57$)$ & 0.011 \\
\hline Duration category (months): & & & \\
\hline Overall & - & - & $<0.001$ \\
\hline $1.01-6.0$ v 0-1 & $-0.12(0.12)$ & $0.88(0.71$ to 1.11$)$ & 0.281 \\
\hline $6.01-12.0$ 0-1 & $-0.51(0.20)$ & $0.60(0.41$ to 0.90$)$ & 0.013 \\
\hline >12 v 0-1 & $-1.17(0.21)$ & $0.31(0.21$ to 0.47$)$ & $<0.001$ \\
\hline Log $_{\mathrm{e}}$ ulcer area & $-0.35(0.05)$ & $0.70(0.64$ to 0.78$)$ & $<0.001$ \\
\hline Recurrent ulceration & $-0.45(0.16)$ & $0.64(0.47$ to 0.87$)$ & 0.005 \\
\hline
\end{tabular}

baseline ulcer area, ulcer duration, and recurrent ulceration are independent predictors of time to healing. ${ }^{21-23}$ Although the effectiveness of the short stretch bandage might be influenced by ankle joint mobility, ${ }^{1}$ we found no significant interaction in our meta-analysis. This is perhaps unsurprising as most patients in the dataset (98\%) were able to walk unaided or with assistance. Previous findings have indicated that the distinction that enables prediction of healing is fixed versus non-fixed joint. ${ }^{21}$

\section{Strengths and weaknesses}

A major strength of this research is the degree of rigour. Our methods were in close accordance with those proposed by the Cochrane Collaboration for conducting systematic reviews of interventions and the Cochrane IPD Meta-analysis Methods Group, the latter being a team who have taken a lead role in developing methods for meta-analyses based on individual patient data. $^{1112}$

All trials used non-blinded assessment of healing. In the largest trial, healing was confirmed from photographs by an investigator blinded to bandage allocation. ${ }^{\text {w1 }}$ Though it is not possible to define the direction or degree of bias that might be present in this meta-analysis from such non-blinded outcome assessment, the potential for this should not be overlooked.

All systematic reviews and meta-analyses can be affected by publication bias. ${ }^{24}$ As neither summary data nor individual patient data were available for the unpublished trial, and we could not generate a hazard

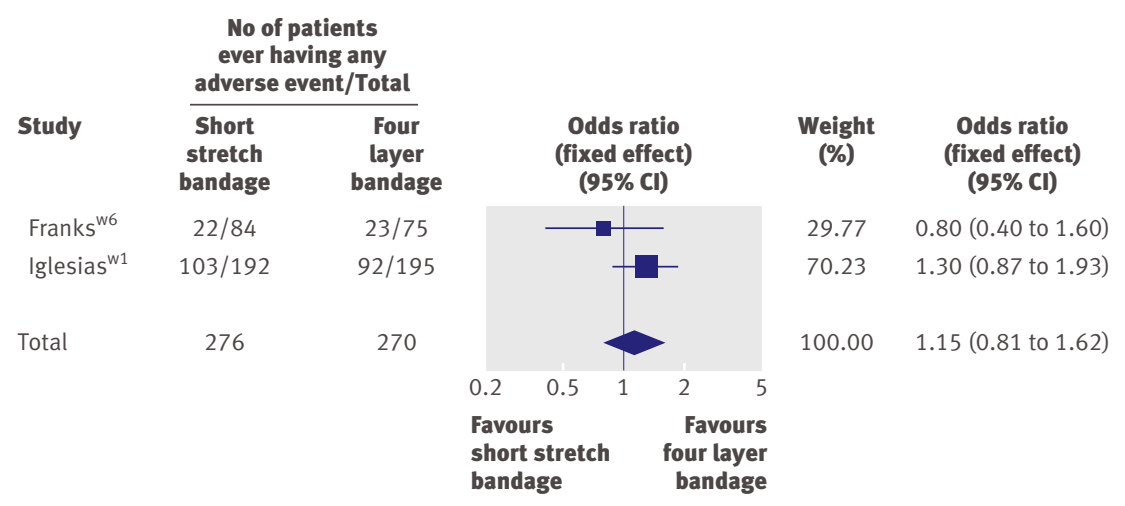

Fig 4 | Pooled odds ratio for incidence of any type of adverse event ratio for the unavailable published trial, ${ }^{\text {w2 }}$ we could not judge the potential effect of including these trials in the meta-analysis. These trials amounted to fewer than $10 \%$ of known randomised patients. While the full dataset is the ideal, the retrieval of around 90\% means that the estimate generated can be viewed with confidence.

The analyses reported in tables 3 and 4 are for fixed effects models; they treated the effect of type of bandage as constant across the trials. We checked this assumption by tests of heterogeneity across trials, testing the interaction between type of bandage and trial. For the data relating to table 3 this gave $\chi^{2}=7.03, \mathrm{df}=4$, and $\mathrm{P}=0.13$. The corresponding statistics for table 4 data were $\chi^{2}=5.84, \mathrm{df}=3$, and $\mathrm{P}=0.12$. For both analyses, this test for heterogeneity reached neither the conventional critical $\mathrm{P}$ value of 0.05 nor the more sensitive critical value of 0.10 sometimes advocated for this test. We were therefore justified in staying with the fixed effect model. When the assumption of a fixed effect is acceptable, introducing random effects might unnecessarily widen confidence intervals and reduce power. As a sensitivity analysis, however, we allowed for a possible random effect of trial by making the trial a cluster and estimating robust standard errors. This approach does not change the estimates but does change their standard errors. For the model in table 3, the hazard ratio for bandage type was 1.29 (95\% confidence interval 1.05 to $1.63 ; \mathrm{P}=0.015)$ and for table 4 was 1.31 ( 1.04 to $1.60 ; \mathrm{P}=0.023)$. Hence, whatever the approach taken we have evidence of a beneficial effect of the four layer bandage.

\section{Implications for further research}

As far as we can ascertain, this is the first example of meta-analysis of individual patient data in wound research. Further such meta-analyses, including both clinical effectiveness and economic data, could be usefully undertaken in the specialty of chronic wound management given that the main outcome is a time to event variable: time to healing.

Further clinical trials could assess related outcomes such as ulcer recurrence and change in ulcer area both as a predictor and as an outcome. In any future trial the area of the ulcer at baseline, the duration of the ulcer, and whether ulceration is recurrent should be used as stratification variables at randomisation, or should be taken into account in the analysis. Primary investigators should provide detailed records of adverse events in compression trials and should aim to arrive at a consensus as to the types of adverse events attributable to the bandage.

The clinical effect of the bandages might in part depend on the skill of the bandager in achieving the correct amount of sub-bandage pressure and pressure graduated from toe to knee. ${ }^{25}$ Consequently, the differential effects of the bandages seen in this meta-analysis could be partly explained by skill and experience of the bandager; three out of the five included trials (representing $75 \%$ of included patients) were based in the UK, where the four layer bandage is standard, ${ }^{\text {w1 w3 w6 }}$ 


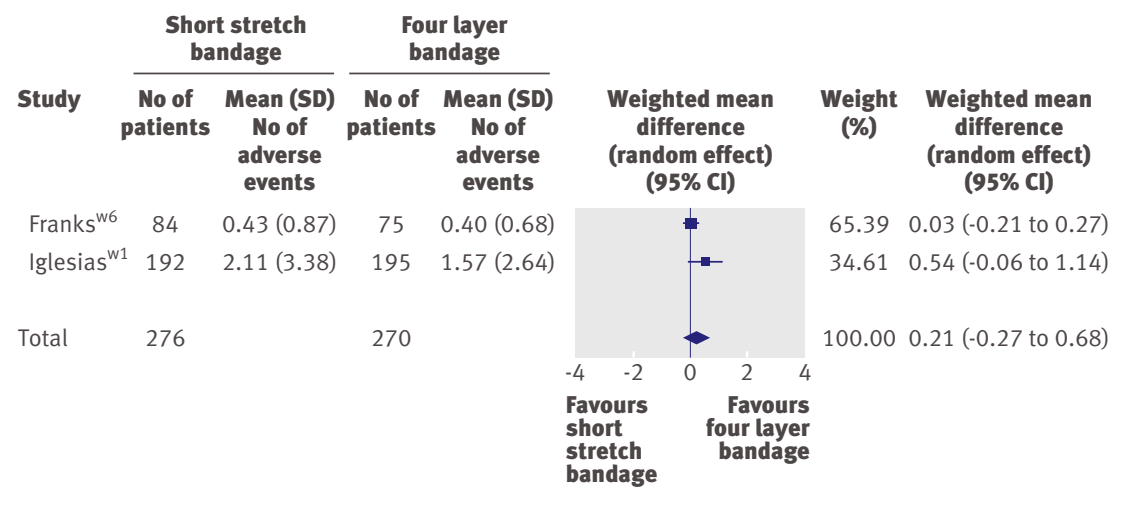

Fig 5 | Pooled effect size for number of adverse events (any type)

while the others were based in continental Europe, where the short stretch bandage is standard treatment. $^{\text {w4 w5 }}$ Information regarding bandager skill was not collected during the included trials and so we could not investigate this further. As far as we could ascertain, the methods used for application of both types of bandage were in line with manufacturers' recommendations, relevant clinical guidelines, and expert guidance. ${ }^{1-27}$ Future trials could usefully collect data on staff skills at baseline, and this information could be included as a covariate in the modelling of treatment effect.

\section{Implications for clinical practice}

Four layer bandaging seems to be more effective than short stretch bandaging in terms of time to healing. Patients with larger ulcers, more chronic ulcers, and recurrent wounds will have longer healing times, regardless of treatment. The findings are likely to be generalisable to most patients with venous leg ulcers who are treated in outpatient clinics or in the community. The choice of bandage system is likely to be influenced by clinician's preference, skill, or familiarity with a locally established regimen and local or national prescribing policies. Other factors influencing the uptake of a treatment include cost and acceptability to patients.

\section{WHAT IS ALREADY KNOWN ON THIS TOPIC}

Venous leg ulceration is a common and recurring condition that imposes a considerable burden on patients and healthcare providers

Compression treatment is the first line treatment, commonly applied as a four layer bandage in the UK and short stretch bandage in other parts of the world

A systematic review of compression based on published trial reports did not detect a difference between the two methods in terms of the number of patients healed at fixed time points

\section{WHAT THIS STUDY ADDS}

When compared with the short stretch bandage, the four layer bandage increases the chance of healing by around $30 \%$ when independent prognostic factors are taken into account

The benefit of the four layer bandage is consistent across patients with differing prognostic profiles, independent predictors of delayed healing being larger baseline ulcer area, more chronic ulceration, and previous ulceration

\section{Conclusions}

Findings suggest that patients with venous leg ulcers treated with four layer bandages experience faster healing than those treated with short stretch bandages. Patients with larger ulcers, older ulcers and recurrent wounds have poorer healing prognosis regardless of treatment. These data suggest, however, that the observed benefits are consistent despite differences in prognosis. Available data from two trials did not suggest a difference in the adverse event profiles of the two bandage types. Further research is required on related outcomes such as ulcer recurrence, change in ulcer area both as a predictor and as an outcome, and cost effectiveness. Future trials should incorporate blinded outcome assessment and, when possible, some assessment of the skill of the bandager at baseline.

We acknowledge the following for their contribution to the original clinical trials included in this meta-analysis: P R F Bell, N J M London, A R Naylor, L E Taylor, A J Wood (University of Leicester); R Blewett and R Martin (Wandsworth Primary Care Trust); J Collins and A Heron (Craigavon Area Hospital Group Trust); R I Damstra (Nij Smallinghe Hospital, Drachten, Netherlands); M J M De Rooii (University Hospital of Nijmegen, Netherlands); A Hildreth, E Seymour (Western Sussex Primary Care Trust); C Hourican (Riverside Primary Care Trust); C P Iglesias, D J Torgerson (University of York); M Konig, A Ukat, W Vanscheidt (University Hospital of Freiberg, Germany); C J Moffatt and M Moody (Thames Valley University); K-C Münter (Dermatology Clinic, Hamburg, Germany); E A Nelson (University of Leeds); D Quinlan (Smith \& Nephew Wound Management); S Schuller-Petrovic (Department of Dermatology, University Clinic, Graz, Austria); D J Tazelaar (Tjongerchans Hospital, Netherlands); R R M Tjon Lim Sang (Leiden, Netherlands); A J Velders (Antonius Hospital, Netherlands). We are most grateful to Professor Hugo Partsch (Wilhelminen Hospital, Vienna) for provision of trial data and valuable advice in interpreting the findings of the meta-analysis. Contributors: SO'M participated in the protocol development, search strategy, data collection, data checking, data analysis, interpretation, and drafting of the manuscript. JT advised on developing the protocol and collecting, checking, and analysing data, and commented on drafts of the manuscript. NC contributed data from the VenUS I trial, participated in protocol development, checked and commented on data analysis, commented on drafts of the manuscript and was joint PhD supervisor for SO'M of whose thesis this work formed a part. JMB advised on the design and statistical analysis and reviewed each draft of the final manuscript. JMB was also joint PhD supervisor for SO'M in his role as professor in the University of York. PJF contributed data, undertook procedures to assure the quality of the raw data, and critically appraised the research work. TM reviewed both draft versions, facilitated the provision of data from the Ukat study, and responded to queries about the data from this study. MS participated in the data collection, submission, verification of accuracy and completeness, and contributed to the manuscript. All authors saw and approved the final version. SO'M is guarantor.

Funding: SO'M is funded by a research scientist award in evidence synthesis from the National Institute for Health Research. The funder had no role in the study design; collection, analysis, and interpretation of data; writing of the report; and the decision to submit the article for publication. Competing interests: NC was principal investigator for the VenUS I trial included in this meta-analysis, for which Beiersdorf provided free trial related bandaging education for trial nurses. The research trial authored by PJF was sponsored by Molnlycke Healthcare and Activa Healthcare. PJF has received research funding in the past from Smith \& Nephew. TM is employed by Smith \& Nephew, who manufacture compression bandages, and he also holds shares in Smith \& Nephew. Ethical approval: Not required.

1 Moffatt C. Compression therapy in practice. Trowbridge: Wounds UK Publishing, 2007.

2 Callam M. Prevalence of chronic leg ulceration and severe chronic venous disease in Western countries. Phlebology 1992;7(suppl 1):6-12.

3 Healthcare Commission. National audit of the management of venous leg ulcers. 2008. www.healthcarecommission.org.uk. 
4 Bosanquet N. Costs of venous ulcers: from maintenance therapy to investment programmes. Phlebology 1992;7(suppl 1):44-6.

5 Ellison DA, Hayes L, Lane C, Tracey A, McCollum CN. Evaluating the cost and efficacy of leg ulcer care provided in two large UK health authorities. J Wound Care 2002;11:47-51.

6 Hamer C, Cullum NA, Roe BH. Patients' perceptions of chronic leg ulcers. J Wound Care 1994;3:99-101.

7 Ebbeskog B, Ekman SL. Elderly persons' experiences of living with venous leg ulcer: living in a dialectal relationship between freedom and imprisonment. Scand J Caring Sci 2001;15:235-43.

8 Morgan PA, Franks PJ, Moffatt C), Doherty DC, O'Connor T, McCullagh L, et al. Illness behaviour and social support in patients with chronic venous ulcers. Ostomy Wound Manage 2004;50:25-32.

9 Cullum N, Nelson EA, Flemming K, Sheldon T. Systematic reviews of wound care management: (5) beds; (6) compression; (7) laser therapy, therapeutic ultrasound, electrotherapy and electromagnetic therapy. Health Technol Assessent 2001;5:1-221.

10 Williams C. Rosidal K: a short-stretch compression bandage system. BrJ Nurs 2001;10:403-4.

11 Stewart LA, Clarke MJ, on behalf of the Cochrane Working Group on Meta-analysis using individual patient data. Practical methodology of meta-analyses (overviews) using updated individual patient data. Stat Med 1995;14:2057-79.

12 Higgins JPT, Green SE, eds. Cochrane handbook for systematic reviews of interventions. Version 5.0.1 (updated September 2008). Oxford: Cochrane Collaboration, 2008. www.cochrane-handbook.org.

13 Altman DG, Bland JM. Units of analysis. BMJ 1997;314:1874.

14 Bland JM, Altman DG. Survival probabilities (the Kaplan-Meier method). BMJ 1998;317:1572.

15 Peduzzi P, Concato J, Kemper E, Holford TR, Feinstein AR. A simulation study of the number of events per variable in logistic regression analysis. J Clin Epidemiol 1996;49:1373-9.
16 Higgins JPT, Thompson SG. Quantifying heterogeneity in a metaanalysis. Stat Med 2002;21:1539-58.

17 Higgins JPT, Thompson SG, Deeks JJ, Altman DG. Measuring inconsistency in meta-analyses. BMJ 2003;327:557-60.

18 Moffatt C, Dorman M. Recurrence of leg ulcers within a community leg ulcer service. J Wound Care 1995;4:56-62.

19 Graham ID, Harrison MB, Nelson EA, Lorimer K, Fisher A. Prevalence of lower-limb ulceration: a systematic review of prevalence studies. Adv Skin Wound Care 2003;16:305-16.

20 Ford I, Norrie J. The role of covariates in estimating treatment effects and risk in long-term clinical trials. Stat Med 2002;21:2899-908.

21 Franks PJ, Moffatt CJ, Connolly M, Bosanquet N, Oldroyd MI, Greenhalgh RM, et al. Factors associated with healing leg ulceration with high compression. Age Ageing 1995;24:407-10.

22 Margolis DJ, Allen-Taylor L, Hoffstad O, Berlin JA. The accuracy of venous leg ulcer prognostic models in a wound care system. Wound Repair Regen 2004;12:163-8.

23 Chaby G, Viseux V, Ramelet AA, Ganry O, Billet A, Lok C. Refractory venous leg ulcers: a study of risk factors. Dermatol Surg 2006;32:512-9.

24 Egger M, Davey Smith G. Meta-analysis: bias in location and selection of studies. BMJ 1998;316:61-6.

25 Feben $\mathrm{K}$. How effective is training in compression bandaging technique? Br J Community Nurs 2003;8:80-4.

26 Rosidal $(R)$ sys. The effective compression therapy of venous ulcers (manufacturer's brochure). Netherlands: Lohmann and Rauscher 2007.

27 Royal College of Nursing. Clinical practice guideline: the nursing management of patients with venous leg ulcers. London: Royal College of Nursing, 2006.

Accepted: 6 March 2009 